Radioactivity in Soils and Sediments in and Adjacent to the Los Alamos Area, 1974-1977

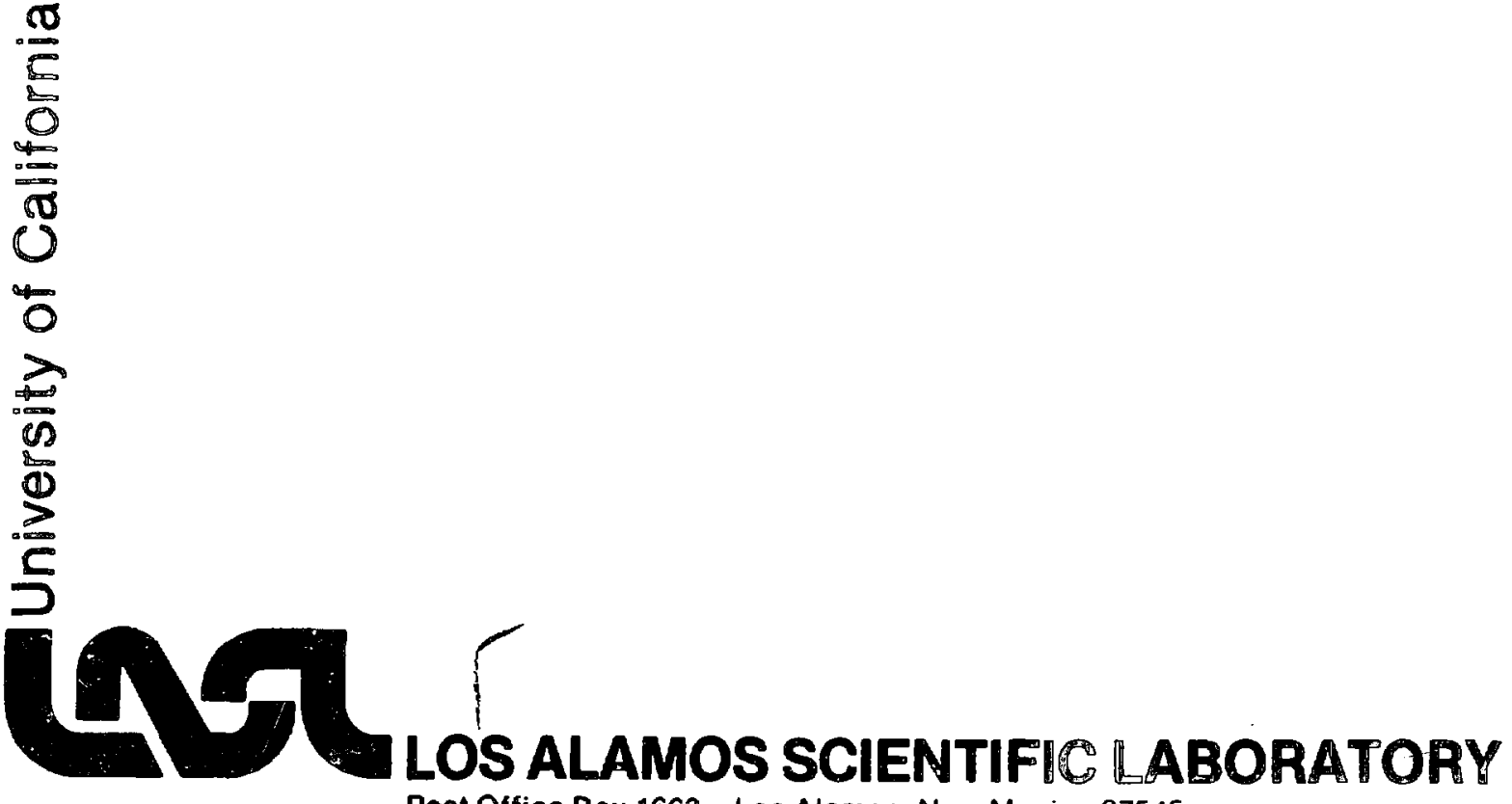




\title{
RADIOACTIVITYIN SOILS AND SEDIMENTS IN AND ADJACENT TO THE LOS ALAMOS AREA, 1974-1977
}

\author{
by
}

\author{
W. D. Purtymun, R. J. Peters, and A. K. Stoker
}

\begin{abstract}
Soil and sediments are analysed for gross alpha, gross beta, ${ }^{238} \mathrm{Pu},{ }^{230} \mathrm{Pu}$, ${ }^{137} \mathrm{Cs}$, ${ }^{00} \mathrm{Sr}$, and total uranium as part of the continuing Environmental Monitoring Program at the Los Alamos Scientific Laboratory. This report documents the leveis of radioactivity of radionuclides in soils and sediments in northern New Mexico from natural sources and worldwide fallout as well as at seven on-site soil and sediment stations which contain radioactivity contributed by the Laboratory for the period 1974 through 1977.
\end{abstract}

\section{INTRODUCTION}

Soils and sediments are collected in and adjacent to the Los Alamos Scientific Laboratory (L.ASL) and analyzed for radioactivity as part of the surveillance program. ${ }^{1.4}$ This study is made annually to determine the effect of the LASL operation on the erivironment. The samples are collected from regionai, perimeter, and on-site stations, which contain radioactivity from natural sources, worldwide fallout from nuclear tests in the atmosphere, and in some cases from effluents released at LASL. The purpose of this report is to document levels of radioactivity in soil and sediments from natural sources and worldwide fallout. Also included in the report for comparison are the analytical data from those stations where LASL has contributed radioactivity to the environment. Locations of the 12 regional soil and sediment stations are shown in Fig. 1. These stations are as much as $45 \mathrm{~km}$ from Los Alamos. Seven perimeter soil and sediments stations are shown in Fig. 2 (adjacent to LASL boundaries). Fourteen on-site soil and sediment stations within the LASL boundaries are also shown in Fig. 2. Other soil and sediment stations have been established for special studies; however, due to the infrequency of samples collected the data from these stations are not included in this report. 5.7

Individual analyses for each station are presented in Appendix I (soil) and Appendix II (sediments) for those stations showing natural and worid-wide fallout levels of radioactivity. Appendix III presents individual analyses for soils, while Appendix IV shows individual analyses of sediments that show elevated radicactivity related to Laboratory releases. The \pm value in the Appendices is twice the analytical error associated with that individual analysis. The Appendices also show minimum, maximum, $\bar{x}$ (the mean), and $s$ (the stan. dard deviation of the distribution of the observed values). 


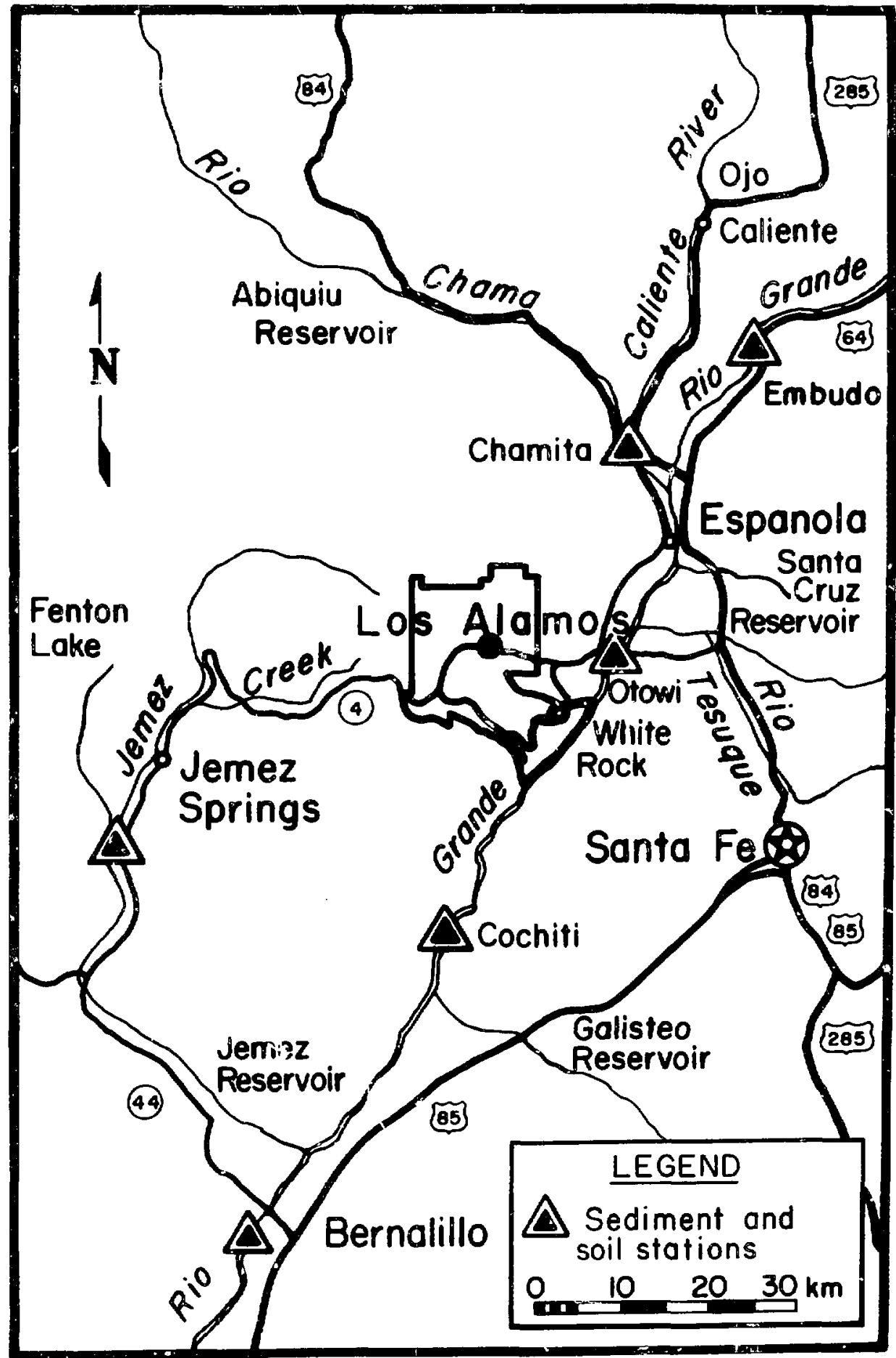

Fig. 1.

Regional soll and sediment stations. 


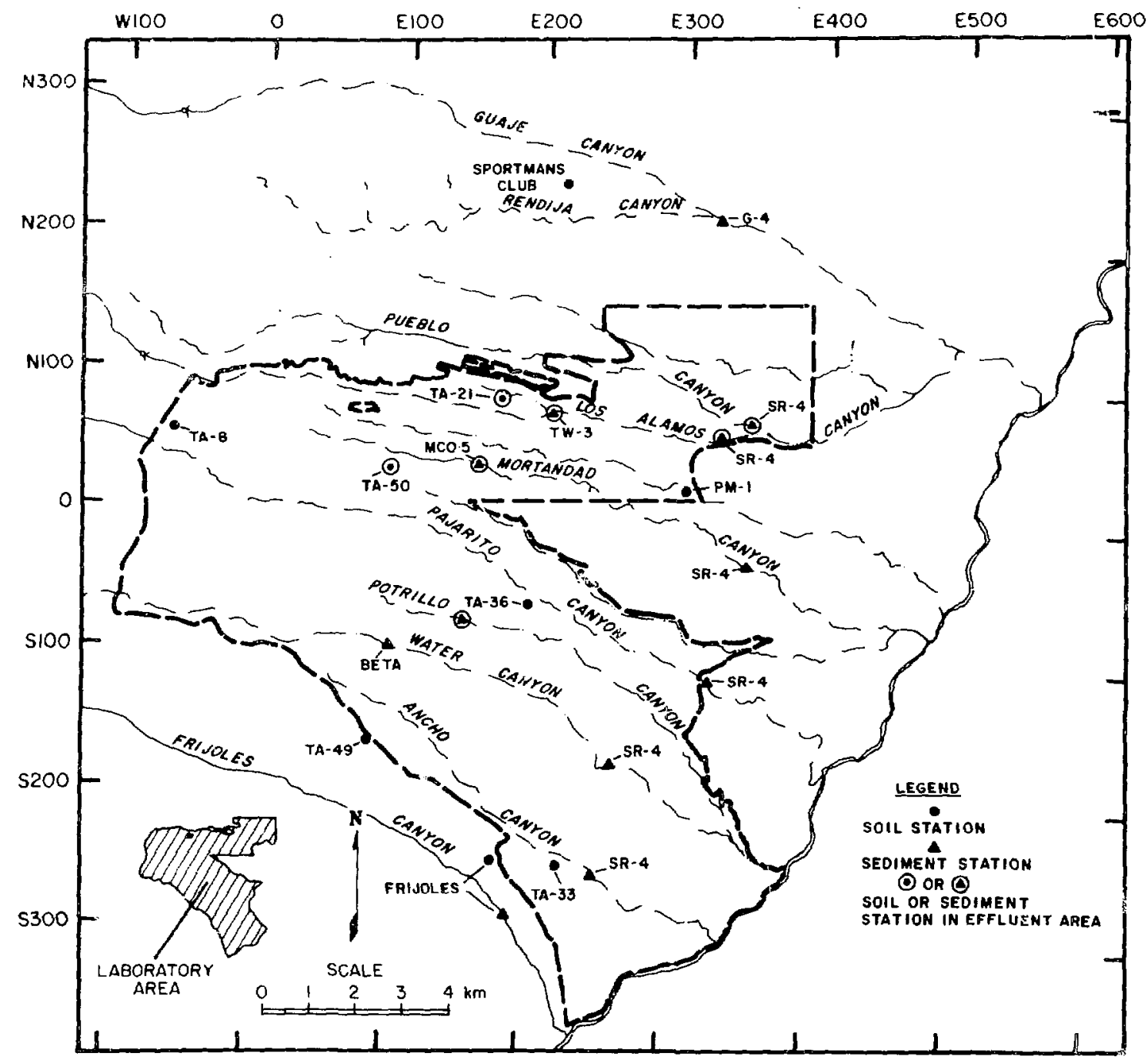

Fig. 2.

Perimeter and on-site soil and sediment stations.

\section{Methods of Sample Collaction and Analysis}

Soil samples were collected by taking five plugs, $7.5 \mathrm{~cm}$ in diameter and $5.0 \mathrm{~cm}$ deep, at the center and corners of a square area $10 \mathrm{~m}$ on a side. The five plugs were combined to form a composite sample for analysis. Sediment samples were collected from dunes behind bculders in the main channels of flowing streams. Samples from the beds of intermittently flowing streams were collected across the main channel to a depth of $\mathbf{5 . 0}$ cm.
The soil and sediment samples were routinely analyzed for gross-alpha ano gross-beta activities and ${ }^{238} \mathrm{Pu}$ and ${ }^{239} \mathrm{Pu}$. Selected samples were also analyzed for ${ }^{19} \mathrm{Cs},{ }^{\circ} \mathrm{Sr}$, and Total $U$. The radionuclides ${ }^{231} \mathrm{Pu},{ }^{238} \mathrm{Pu},{ }^{137} \mathrm{Cs}$, and ${ }^{\circ 0} \mathrm{Sr}$ are artifically created. Gross alpha, gross beta, and total uranium generally reflect naturally occurring radioactivity. The addition of radionuclides from fallout is so low that they are not reflected in the gross alpha and beta activity. Total uranium is generally associated with naturally occurring uranium minerals found in the soil and sediments. 
Only in the effluent release areas of the Laboratory do the gross beta and total uranium reflect the contributions from the Laboratory's operation.

A brief description is presented of sample preparation and analysis. A detailed description of these procedures is presented elsewhere."

Soil and sediment samples are oven dried, sieved through $<1.7 \mathrm{~mm}$ screens and thoroughly blended. Separate aliquots of the prepared soil are taken for each analysis. Soil for gross alpha and beta analysis is leached with hot acid and the leachate is evaporated onto a stainless steel planchet prior to counting in a thin window, dual gas proportional counter. Soil for ${ }^{238.230} \mathrm{Pu}$ analysis is spiked with ${ }^{242} \mathrm{Pu}$ tracer and dissolved with acid. The $\mathrm{Pu}$ is isolated by anion exchange, electroplated onto a stainless steel disk, and counted on an alpha spectrometer. Soll for ${ }^{137} \mathrm{Cs}$ analysis is counted directly on $\mathrm{Ge}(\mathrm{Li})$ detector coupled with a multichannel analyzer. Soil for ${ }^{\circ 0} \mathrm{Sr}$ analysis is dissolved. The sample is extracted with HDEHP to remove the ${ }^{90} Y$ daughter and any interferring radionuclides. Siable $Y$ is added as a chemical carrier. After allowing the ${ }^{00} Y$ daughter to reequilibrate with the ${ }^{\circ 0} \mathrm{Sr}$, the sample is reextracted, the $Y$ is purified, and the sample counted on a gas proportional counter. The total uranium analyses from 1974 through 1976 were performed using a fluorometric method. In 1977 , aliquots of soil for uranium analysis were irradiated by epithermal neutrons in a reactor and then counted on a $\mathrm{Ge}(\mathrm{Li})$ gamma ray spectrometer.

The minimum detection limits are as follows:

$\begin{array}{ll}\text { Gross Alpha } & 0.8 \mathrm{pCi} / \mathrm{g} \\ \text { Gross Beta } & 0.0003 \mathrm{pCi} / \mathrm{g} \\ { }^{238} \mathrm{Pu} & 0.003 \mathrm{pCi} / \mathrm{g} \\ { }^{239} \mathrm{Pu} & 0.002 \mathrm{pCi} / \mathrm{g} \\ { }^{137} \mathrm{Cs} & 0.10 \mathrm{pCi} / \mathrm{g} \\ { }^{00} \mathrm{Sr} & 0.05 \mathrm{pCi} / \mathrm{g} \\ \text { Total U } & 0.1 \mu \mathrm{g} / \mathrm{g}\end{array}$

The anaiyses, particularly hose for which a chemical or instrument background must be subtracted, may result in a net value that is lower than the minimum detection limits. It is not uncommon for individual analysis to result in values of zero or negative numbers. The negative value does not represent a physical reality; however, a valid longterm average of many analyses can be obtained only if the very small or negative values are included in the mean.

\section{RADIOACTIVITY FROM NATURAL SOURCES AND WORLDWIDE FALLOUT}

Soil and sediment analyses from 25 soil and sediment stations were collected once or twice annually from 1974 through 1977 . The number of analyses from the stations ranged from 68 for ${ }^{\circ 0} \mathrm{Sr}$ to 153 for gross alpha and gross beta (Table I).

The average gross alpha concentration for soil was slightly higher $(4.9 \pm 3.4 \mathrm{pCi} / \mathrm{g})$ than sediments $(3.3 \pm 3.2 \mathrm{pCi} / \mathrm{g})$. The levels of gross alpha for the 153 soil and sediment analyses ranged from 0.1 to $18 \mathrm{pCi} / \mathrm{g}$ with an overall average of $4.0 \pm 3.2$ pCi/g (Table I).

The average gross beta concentration for soils was higher $(6.7 \pm 2.4 \mathrm{pci} / \mathrm{g})$ than sediments $(3.7 \pm$ $2.8 \mathrm{pCi} / \mathrm{g}$ ). The levels of gross beta for the 153 soil and sediment analyses ranged from 0.2 to $13 \mathrm{pCi} / \mathrm{g}$ with an overall average of $5.2 \pm 3 \mathrm{pCi} / \mathrm{g}$ (Table 1 ).

The ${ }^{238} \mathrm{Pu}$ levels in both soils and sediments were near or below the limits of detection. The average concentration for soils was $0.000 \pm 0.004 \mathrm{pCi} / \mathrm{g}$ while that of sediments was also $0.000 \pm 0.003$ $\mathrm{pCi} / \mathrm{g}$. The range of 151 analyses of both soils and sediments was from -0.030 to $0.010 \mathrm{pCi} / \mathrm{g}$ with an overall average $0.000 \pm 0.004 \mathrm{pCi} / \mathrm{g}$.

The average coricentration of ${ }^{230} \mathrm{Pu}$ on soil $(0.012$ $\pm 0.012 \mathrm{pCi} / \mathrm{g}$ ) was higher than that for sediments $(0.004 \pm 0.007 \mathrm{pCi} / \mathrm{g})$. The rangt of 149 analyses was -0.020 to $0.045 \mathrm{pCi} / \mathrm{g}$, with an overall average of $0.008 \pm 0.010 \mathrm{pCi} / \mathrm{g}$ (Table I).

The ${ }^{137} \mathrm{Cs}$ level in soils $(0.52 \pm 0.30 \mathrm{pCi} / \mathrm{g})$ was higher than in sediments $(0.17 \pm 0.19 \mathrm{pCi} / \mathrm{g})$. The 76 analyses ranged from -0.14 to $1.06 \mathrm{pCi} / \mathrm{g}$ with an overall average of $0.32 \pm 0.30 \mathrm{pCi} / \mathrm{g}$ (Table 1 ).

The ${ }^{90} \mathrm{Sr}$ average concentrations on suils $(0.33 \pm$ $0.27 \mathrm{pCi} / \mathrm{g})$ were higher than on sediments $(0.20 \pm$ $0.25 \mathrm{pCi} / \mathrm{g}$ ). The range of ${ }^{\circ 0} \mathrm{Sr}$ for the 68 analyses of soils and sediments was from -0.01 to $1.00 \mathrm{pCi} / \mathrm{g}$ with an overall average of $0.25 \pm 0.27 \mathrm{pCi} / \mathrm{g}$.

The average values of gross alpha, gross beta, ${ }^{230} \mathrm{Pu},{ }^{239} \mathrm{Pu},{ }^{137} \mathrm{Cs}$, arid ${ }^{\circ 0} \mathrm{Sr}$ are lower in ingdiments than in soils as the sediments in part sen be derived by erosion from materials not expesed tc world-wide fallout. 
TABLE I

\section{SUMMARY OF NATURAL OR WORLDWIDE FALLOUT LEVELS OF RADIOACTIVITY OR RADIONUCLIDES \\ (Analyses in $\mathrm{pCi} / \mathrm{g}$ except as noted)}

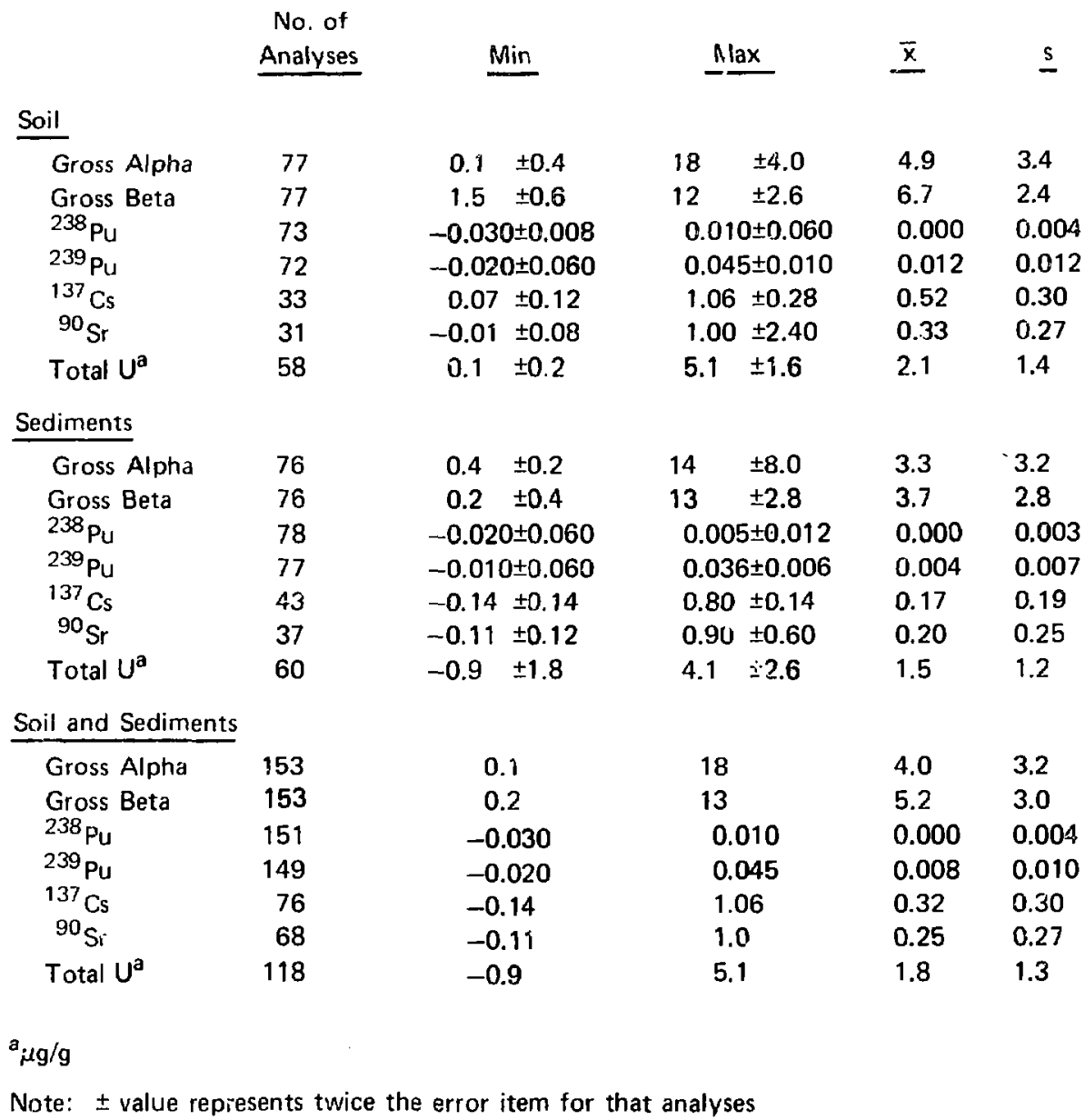

The total uranium in the regional soils and sediments results mainly from natural occurrence of uranium. Trace amounts may be attributed to worldwide fallout but are too low to distinguish. The total uranium content of soils or sediments from individual stations will vary because of chemical or mechanical weathering of different parent materials that contain varying amounts of uranium.

The average total uranium was slightly higher in soils $(2.1 \pm 1.4 \mu \mathrm{g} / \mathrm{g})$ than in sediments $(1.5 \pm 1.2$ $\mu \mathrm{g} / \mathrm{g}$ ). The range of total uranium of from 118 soil and sediment analyses ranged from $-C .9$ to 5.1 $\mu \mathrm{g} / \mathrm{g}$ with an overall average of $1.8 \pm 1.3 \mathrm{ug} / \mathrm{g}$ (Table 1).

\section{LeVELS OF RADBONUCLIDES RELATED to RELEASE OF INDUSTRIAL EFFLLENTS}

For the purpose of this report, individual sample results are considered above background (natural and worldwide fallout levels for Northern New Mexico) if they exceed the mean and 2 times the standard deviation (Table 1). The mean plus the 2 times 
the standard deviation includes approximately $95 \%$ of the population of samples. Two soil and five sediment stations from routine surveillance stations have shown levels of radionuclides in excess of background. The stations are in effluent release areas for industrial wastes. They are within the LASL boundaries (Fig. 2).

\section{A. Soil from Effluent Areas}

Samples from two stations within the LASL boundaries contained levels of plutonium in excess of fallout levels of radioactivity (Appendix III). Technical Area 21 (TA-21) is the site of a plutonium processing plant and also contains a liquid radioactive waste treatment plant. Technical Area 50 (TA-50) is the site of the major liquid radioactice waste treatment plant (Fig. 2). Some radioactivity is vented to the atmosphere at both areas after passing through filters which retain most of the radioactive particles.

Only two of the five samples of soil collected at TA-21 contained plutonium in excess of worldwide fallout. Both samples were collected in 1976. The major isotope was ${ }^{239} \mathrm{Pu}$ (Table II).

All five of the samples collected at TA-50 since 1975 have contained piutonium in excess of fallout levels (Table II). The major isotope is ${ }^{230} \mathrm{Pu}$. The largest concentrations were in samples collected in 1977.

\section{B. Sediments from Effluent Areas}

Samples from four sediment stations downgradient from liquid treatment plants contained higher levels of radionuclides when compared to those canyons that do not receive liquid effluents (Appendix IV). The concentrations of nuclides in sediments have varied because of transport and dispersion of contaminated sediments by storm runoff.

The flow in the canyons is intermittent, from effluent release and storm runoff. Only during periods of heavy storm runoff does the runoff reach the Rio Grande in Pueblo and Los Alamos Canyons. There has been no runoff outside the LASL boundaries in Mortandad Canyon since 1960. Effluents were first released into the canyon in $1963 .^{1}$

Sediments from Pueblo Canyon at SR-4 contain above background levels of plutcnium as the canyon received effluents until 1964. The major isotope is ${ }^{239} \mathrm{Pu}$ (Table III). The concentrations have varied slightly but have essentially remained the same during the period 1975 through 1977 indicating that, at the present, transport and dispersion in the lower reach of the canyon have reached equilibrium.

Samples from two on-site sediment stations in Los Alamos Canyon contained above background levels of radioactivity. The canyon has received treated liquid radioactive effluents from the treatment plant at TA-21 since 1952.

TABLE II

\author{
SUMMARY OF PLUTONIUM ANALYSES OF SOIL FROM \\ EFFLUENT RELEASE AREAS \\ (Analyses in pCi/g)
}

$\begin{array}{ccccccc} & \begin{array}{c}\text { No. of } \\ \text { Analyses }\end{array} & \text { Min } & & \text { Max } & \underline{\bar{x}} & \underline{s} \\ & & & & & \\ 238 \mathrm{Pu} & 5 & 0.000 & 0.008 & 0.004 & 0.003 \\ 239 \mathrm{Pu} & 5 & 0.005 & 0.600 & 0.167 & 0.254 \\ & & & & & \\ \text { TA-50 } & & & & & \\ 238 \mathrm{Pu} & 5 & 0.003 & 0.017 & 0.006 & 0.006 \\ 239 \mathrm{Pu} & 5 & 0.088 & 6.98 & 1.607 & 3.014\end{array}$


TABLE III

\section{SUMMARY OF RADIOCHEMICAL ANALYSES OF SEDIMENT \\ FROM EFFLUENT RELEASE AREAS \\ (Analyses in pCi/g except as noted)}

\begin{tabular}{|c|c|c|c|c|c|}
\hline & $\begin{array}{c}\text { No. of } \\
\text { Analyses } \\
\end{array}$ & Min & Max & $\bar{x}$ & $\underline{s}$ \\
\hline \multicolumn{6}{|c|}{ Pueblo at SR -4} \\
\hline $238 \mathrm{r} u$ & 6 & 0.000 & 0.003 & 0.002 & 0.001 \\
\hline${ }^{239} \mathrm{Pu}$ & 6 & 0.011 & 0.380 & 0.267 & 0.138 \\
\hline \multicolumn{6}{|c|}{ Los Alamos at TW-3 } \\
\hline${ }^{238} \mathrm{Pu}$ & 6 & 0.002 & 0.115 & 0.070 & 0.046 \\
\hline${ }^{239} \mathrm{Pu}$ & 6 & 0.040 & 0.720 & 0.274 & 0.272 \\
\hline${ }^{137} \mathrm{Cs}$ & 4 & 0.16 & 26 & 13 & 15 \\
\hline${ }^{90} \mathrm{Sr}$ & 4 & 2.4 & 8.5 & 4.9 & 2.6 \\
\hline \multicolumn{6}{|c|}{ Los Alamos at SR -4} \\
\hline${ }^{238} \mathrm{Pu}$ & 6 & -0.003 & 0.008 & 0.003 & 0.004 \\
\hline${ }^{239} \mathrm{Pu}$ & 6 & 0.021 & 0.163 & $0.05 !$ & 0.055 \\
\hline \multicolumn{6}{|c|}{ Mortandad at $\mathrm{MCO}-5$} \\
\hline${ }^{238} \mathrm{Pu}$ & 5 & 4.07 & 6.01 & 4.83 & 0.74 \\
\hline${ }^{239} \mathrm{P}_{\mathrm{L}}$ & 5 & 0.95 & 15.6 & 3.91 & 6.54 \\
\hline${ }^{137} \mathrm{Cs}$ & 2 & -- & 71 & 71 & 0 \\
\hline${ }^{90} \mathrm{Sr}$ & 1 & -- & 2.7 & -- & $-\rightarrow$ \\
\hline \multicolumn{6}{|c|}{ Potrillo Canyor at T A-36 } \\
\hline Total $U^{\bullet}$ & 4 & 0.7 & 5.6 & 3.6 & 2.3 \\
\hline
\end{tabular}

Los Alamos Canyon at TW-3 is about $1.2 \mathrm{~km}$

The sediment station in Potrillo Canyon at TA-36 below the outfall from the treatment plant. Gross beta activity, ${ }^{238} \mathrm{Pu},{ }^{230} \mathrm{Pu},{ }^{137} \mathrm{Cs}$, and ${ }^{80} \mathrm{Sr}$ have been above background levels at verious times during the period 1975-77 (Table Hi). The levels have varied from sample to sample reflecting transport and dispersion with storm runoff.

Los Alamos Canyon at SR-4 is located about 3.6 $\mathrm{km}$ downgradient from the station at TW-3 (Fig. 2). During the collection period (1975-77), all samples contained ${ }^{238} \mathrm{Pu}$ above background. Radionuclides in sediments at TW-3 have been dispersed by storm runoff so that lower concentrations occur at SR-4.

Mortandad Canyon at MCO-5 is $2.2 \mathrm{~km}$ below the outfall from the liquid radioactive waste treatment plant at TA-50. The sedimsints have contained gross beta, ${ }^{236} \mathrm{Pu},{ }^{230} \mathrm{Pu},{ }^{137} \mathrm{Cs}$, and ${ }^{90} \mathrm{Sr}$ levels that were above background. The concentrations of ${ }^{238} \mathrm{Pu}$ and ${ }^{238} \mathrm{Pu}$ have varied, however, ${ }^{230} \mathrm{Pu}$ was the major isotope of plutonium released in the past few years. lies downgradient from a test area that expends some uranium. Uranium was background in 1975 and no samples were analyzed in 1976. The total uranium in 1977 was slightly above background at 5.6 and $5.1 \mu \mathrm{g} / \mathrm{g}$. The 1977 results indicate some transport by storm runoff from the test area into the canyon.

\section{SUMMARY}

The gross alpha, gross beta, and total uranium reflect natural occurring radioactivity in the rock forming minerals in the soil and sediments. The levels of gross alpha, gross beta, and total uranium added to the soils and sediments from fallout are too low to distinguish from the higher levels that occurred from the natural radioactivity. The fallout levels of ${ }^{230} \mathrm{Pu},{ }^{230} \mathrm{Pu},{ }^{137} \mathrm{Cs}$, and ${ }^{00} \mathrm{Sr}$ are evident in the soils and sediments. The mean values of ${ }^{238} \mathrm{Pu}$, ${ }^{239} \mathrm{Pu},{ }^{137} \mathrm{Cs}$, and ${ }^{\circ 0} \mathrm{Sr}$ are lower in the sediments 
than in the soils as sediments in part are derived by erosion from materials not exposed to world-wide fallout.

The radioactivity attributed to release of industrial effluents was found in samples from two soit and five sediment stations. The levels of radionuclides varied, showing no particular trends due to dispersion and transport by storm runoff.

\section{REFERENCES}

1. K. E. Apt and V. J. Lee, Compilers, "Environmental Surveillance at Los Alamos During 1974," Los Alamos Scientific Laboratory report LA-5977-PR (1975).

2. K. E. Apt and V. J. Lee, Compilers, "Environmental Surveillance at Los Alamos During 1975." Los Alamos Scientific Laboratory report LA-6321-MS (1976).

3. Environmental Studies Group, "Environmental Surveillance at Los Alamos During 1976," Los Alamos Scientific Laboratory report LA-6301MS (1977).
4. Environmental Surveillance Group, "Environmental Surveillance at Los Alamos During 1977," Los Alamos Scientific Laboratory report LA-7263-MS (1978).

5. W. D. Purtymun, "Plutonium in Stream Channel Alluvium in the Los Alamos Area," Los Alamos Scientific Laboratory report LA-4561 (1971).

6. W. R. Kennedy and W. D. Purtymun, "Plutonium and Strontium in Soil in the Los Alamos, Espanola, and Sarita Fe Areas, New Mexico," Los Alamos Scieritific Laboratory report LA4562 (1971).

7. W. R. Kennedy and W. D. Purtymun, "Plutonium and Strontium in Soil Near Technical Area 21, Los Alamos Scientific Laboratory, Los Alamos, New Mexico," Los Alamos Scientific Laboratory report LA-4563 (1971).

8. W. C. Hansen and F. R. Miera, Jr., "Continued Studies of Long-Term Ecological Effects of Exposure to Uranium," Los Alamos Scientific Laboratory report LA-6742 (1977). 
APPENDIX!

RADIOCHEMICAL ANALYSES OF SOILS SHOWING NATURAL OR WORLDWIDE-FALLOUT LEVELS

$\mathrm{pCi} / \mathrm{g}$ Date

Regional

Chamita

\section{$7 \cdot 1474$}

$5-8-75$
$10-7-75$

32976

10.4-76

$3.7-77$

$10-18-77$

7.1474

5-8-75

10-7-75

$29-76$

$10-4-76$

3.7.77

10-18-77

$7-14-74$

5-9-75

10-4-76

3-8-77

10.1477

Cochiti

5-8.75

10-9-75

$42-76$

10-5-76

$3-877$

10-19-77

Bernalillo

\begin{tabular}{|c|c|c|c|c|c|c|}
\hline \multicolumn{6}{|c|}{$\mathrm{pCi} / 9$} & \multirow{2}{*}{$\begin{array}{l}\mu \mathrm{g} / 9 \\
\text { Total U } \\
\end{array}$} \\
\hline Gross Alpha & Gross Beta & ${ }^{2,28} \mathrm{P}_{u}$ & ${ }^{239} \mathrm{Pu}_{\mathrm{u}}$ & ${ }^{137} \mathrm{Cs}_{\mathrm{S}}$ & ${ }^{90} \mathrm{Sr}$ & \\
\hline $20 \pm 0.6$ & $9.7 \pm 1.0$ & $0.001 \pm 0.002$ & $0.006 \pm 0.004$ & $0.81 \pm 0.26$ & - & - \\
\hline $2.9 \pm 1.2$ & $5.7 \pm 2.4$ & - & - & - & - & $0.8 \pm 0.2$ \\
\hline $3.4 \pm 1.6$ & $5.1 \pm 1.2$ & $0.000 \pm 0.001$ & $0.017 \pm 0.006$ & - & - & $0.8 \pm 0.2$ \\
\hline $6.1 \pm 3.0$ & $7.4 \pm 1.6$ & $0.001 \pm 0.001$ & $0.008 \pm 0.003$ & $0.68 \pm 0.06$ & - & $2.1 \pm 0.8$ \\
\hline $1.2 \pm 1.2$ & $5.2 \pm 1.2$ & $0.002 \pm 0.004$ & $0.014 \pm 0.004$ & - & $0.38 \pm 0.32$ & - \\
\hline $10 \pm 4.0$ & $7.7 \pm 1.8$ & $-0.002 \pm 0.002$ & $0.017 \pm 0.006$ & $0.75 \pm 0.12$ & $0.09 \pm 0.14$ & $3.0 \pm 1.8$ \\
\hline $3.9 \pm 2.0$ & $8.2 \pm 2.0$ & $-0.001 \pm 0.000$ & $0.010 \pm 0.0066$ & $0.57 \pm 0.16$ & $0.29 \pm 0.18$ & $3.0 \pm 1.8$ \\
\hline $0.7 \pm 0.4$ & $11 \pm 1.4$ & $0.001 \pm 0.002$ & $0.005 \pm 0.002$ & $0.56 \pm 0.26$ & - & - \\
\hline $2.8 \pm 1.2$ & $5.7 \pm 2.4$ & $0.000 \pm 0.002$ & $0.008 \pm 0.004$ & - & - & $1.2 \pm 0.4$ \\
\hline $2.0 \pm 1.0$ & $3.9 \pm 1.0$ & $0.000 \pm 0.001$ & C.009 \pm 0.003 & - & - & $0.1 \pm 0.2$ \\
\hline $5.0 \pm 2.4$ & $8.3 \pm 1.8$ & $0.001 \pm 0.002$ & $0.012 \pm 0.004$ & $0.77 \pm 0.06$ & $0.90 \pm 2.00$ & $1.1 \pm 9.6$ \\
\hline $3.5 \pm 1.8$ & $5.3 \pm 1.4$ & $0.003 \pm 0.003$ & $0.019 \pm 0.006$ & - & - & - \\
\hline $3.9 \pm 2.0$ & $5.9 \pm 1.4$ & $0.001 \pm 0.010$ & $0.010 \pm 0.010$ & $0.37 \pm 0.06$ & $0.40 \pm 0.22$ & $2.2 \pm 1.6$ \\
\hline $4.3 \pm 2.2$ & $8.0 \pm 1.8$ & $-0.002 \pm 0.004$ & $0.015 \pm 0.006$ & $0.72 \pm 0.16$ & - & $1.8 \pm 1.2$ \\
\hline $0.8 \pm 0.4$ & $8.3 \pm 0.8$ & $-0.002 \pm 0.008$ & $0.012 \pm 0.008$ & $0.76 \pm 0.26$ & - & - \\
\hline $2.7 \pm 1.2$ & $5.2 \pm 2.2$ & $0.002 \pm 0.003$ & $0.006 \pm 0.004$ & - & - & $1.3 \pm 0.4$ \\
\hline $2.7 \pm 1.4$ & $4.4 \pm 1.2$ & - & - & - & - & - \\
\hline $5.8 \pm 2.8$ & $7.2 \pm 1.6$ & - & - & $1.06 \pm 0.28$ & $0.04 \pm 0.16$ & $3.7 \pm 2.0$ \\
\hline $27 \pm 1.4$ & $5.6 \pm 1.4$ & $-0.001 \pm 0.001$ & $0.021 \pm 0.006$ & $0.95 \pm 0.28$ & $0.20 \pm 0.20$ & $3.9 \pm 1.8$ \\
\hline $3.1 \pm 1.4$ & $6.1 \pm 2.6$ & $0.000 \pm 0.002$ & $0.002 \pm 0.003$ & - & - & $1.0 \pm 0.2$ \\
\hline $4.0 \pm 2.0$ & $5.3 \pm 1.2$ & $0.001 \pm 0.002$ & $0.000 \pm 0.000$ & - & - & $1.6 \pm 0.2$ \\
\hline $12 \pm 6.0$ & $8.7 \pm 2.0$ & $0.000 \pm 0.001$ & $0.004 \pm 0.002$ & - & $1.00 \pm 2.40$ & $1.4 \pm 0.6$ \\
\hline $28 \pm 1.8$ & $3.3 \pm 1.0$ & $0.001 \pm 0.002$ & $0.003 \pm 0.003$ & - & - & - \\
\hline $10 \pm 6.0$ & $7.2 \pm 1.6$ & $-0.002 \pm 0.003$ & $0.001 \pm 0.004$ & $0.12 \pm 0.18$ & $0.03 \pm 0.06$ & $1.9 \pm 1.6$ \\
\hline $3.9+1.8$ & $6.0 \pm 1.4$ & $0.001 \pm 0.003$ & $0.003 \pm 0.004$ & $0.48 \pm 0.10$ & $0.34 \pm 0.22$ & $2.3 \pm 1.2$ \\
\hline $0.6 \pm 0.4$ & $8.7 \pm 0.8$ & $0.000 \pm 0.001$ & $0.006 \pm 0.004$ & $0.33+0.24$ & - & - \\
\hline $2.3 \pm 1.0$ & $4.4 \pm 1.8$ & $0.001 \pm 0.002$ & $0.012 \pm 0.005$ & - & - & $0.6 \pm 0.2$ \\
\hline $6.0 \pm 3.0$ & $5.7 \pm 1.4$ & $0.000 \pm 0.001$ & $0.001 \pm 0.001$ & - & - & $1.9 \pm 1.2$ \\
\hline $3.4 \pm 1.8$ & $3.7 \pm 1.0$ & $0.000 \pm 0.001$ & $0.004 \pm 0.002$ & $0.24 \pm 0.04$ & - & $0.9 \pm 0.6$ \\
\hline $1.5 \pm 1.2$ & $3.3 \pm 1.0$ & $0.002 \pm 0.002$ & $0.002 \pm 0.002$ & - & - & - \\
\hline $4.6 \pm 2.6$ & $4.6 \pm 1.2$ & $-0.002 \pm 0.012$ & $-0.002 \pm 0.010$ & $0.06 \pm 0.04$ & $0.05 \pm 0.03$ & $2.7 \pm 2.0$ \\
\hline $5.2 \pm 2.6$ & $5.8 \pm 1.6$ & $0.000 \pm 0.001$ & $0.002 \pm 0.004$ & $0.32 \pm 0.06$ & $0.24 \pm 0.20$ & $2.1 \pm 1.2$ \\
\hline $0.1 \pm 0.4$ & $9.9 \pm 1.0$ & $0.000 \pm 0.000$ & $0.001 \pm 0.004$ & $0.11 \pm 0.24$ & - & - \\
\hline $2.2 \pm 1.0$ & $4.4 \pm 1.8$ & $-0.002 \pm-0.002$ & $0.001 \pm 0.003$ & - & - & $0.5 \pm 0.1$ \\
\hline $1.4 \pm 0.8$ & $2.5 \pm 0.8$ & $-0.001 \pm-0.002$ & $0.012 \pm 0.005$ & - & - & $0.1 \pm 0.1$ \\
\hline $7.2 \pm 3.6$ & $5.7 \pm 1.4$ & $0.001 \pm 0.002$ & $0.002 \pm 0.002$ & $0.11 \pm 0.03$ & $0.98 \pm 2.40$ & $1.1+0.6$ \\
\hline $1.5 \pm 1.6$ & $3.6 \pm 1.0$ & $0.001 \pm 0.002$ & $0.007 \pm 0.004$ & $0.14 \pm 0.04$ & $0.05 \pm 0.40$ & - \\
\hline $15 \pm 8.0$ & $8.6 \times 2.0$ & $0.010 \pm 0.060$ & $-0.020 \pm 0.060$ & $0.21 \pm 0.10$ & $0.14 \pm 0.08$ & $1.7 \pm 1.8$ \\
\hline $1.7 \pm 1.2$ & $5.4 \pm 1.4$ & $0.000 \pm 0.004$ & $0.001 \pm 0.004$ & $0.07 \pm 0.12$ & $0.26 \pm 0.22$ & $1.2 \pm 1.2$ \\
\hline
\end{tabular}


APPENOIX I

RADIOCHEMICAL ANALYSES OF SOILS SHOWING NATURAL OR WORLDWIDE-FALLOUT LEVELS (continued)

\begin{tabular}{|c|c|c|c|c|c|c|c|}
\hline \multirow[b]{2}{*}{ Date } & \multicolumn{6}{|c|}{$\mathrm{pCs} / \mathrm{g}$} & \multirow{2}{*}{$\begin{array}{l}\mu g / g \\
\text { Total U }\end{array}$} \\
\hline & Gross Appha & Gross Bote & ${ }^{236} \mathrm{Py}$ & ${ }^{239} \mathrm{Pu}$ & ${ }^{137} a$ & ${ }^{90} \mathrm{Sr}$ & \\
\hline No. of Analysis & 39 & 39 & 36 & 36 & 22 & 16 & 28 \\
\hline Minimum & 0.1 & 2.5 & -0.002 & -0.020 & 0.07 & 0.03 & 0.1 \\
\hline Maximum & 15 & 11 & 0.010 & 0.019 & 1.06 & 1.00 & 3.9 \\
\hline $\bar{x}$ & 4.0 & 6.2 & 0.000 & 0.006 & 0.46 & 0.34 & 1.6 \\
\hline s & 3.2 & 2.0 & 0.002 & 0.007 & 0.31 & 0.33 & 1.0 \\
\hline
\end{tabular}

\begin{tabular}{|c|c|c|c|c|c|c|c|c|}
\hline \multicolumn{9}{|l|}{ Perimoter } \\
\hline \multirow[t]{6}{*}{ Sportsman Club } & $5-12.75$ & $4.8 \pm 2.0$ & $8.3 \pm 3.4$ & $0.002 \pm 0.002$ & $0.022 \pm 0.006$ & - & - & $0.1 \pm 0.2$ \\
\hline & $10-6.75$ & $3.1 \pm 1.4$ & $9.8 \pm 2.2$ & $0.002 \pm 0.002$ & $0.042 \pm 0.007$ & - & - & $0.1 \pm 0.2$ \\
\hline & 4.21 .76 & $3.7 \pm 1.6$ & $3.4 \pm 0.8$ & $0.002 \pm 0.002$ & $0.012 \pm 0.004$ & - & - & $2.5 \pm 0.8$ \\
\hline & $10-13-76$ & $3.9+1.8$ & $5.3+1.4$ & $0.004 \pm 0.003$ & $0.021 \pm 0.006$ & - & - & - \\
\hline & $4-12.77$ & $9.0 \pm 4.0$ & $8.4 \pm 2.0$ & $-0.003 \pm 0.008$ & $0.022 \pm 0.016$ & $1.04 \pm 0.18$ & $-0.01 \pm 0.08$ & $4.0 \pm 2.2$ \\
\hline & $10-14-77$ & $5.7 \pm 2.6$ & $8.7 \pm 2.0$ & $-0.903 \pm 0.003$ & $0.015 \pm 0.006$ & $0.81 \pm 0.18$ & $0.50 \pm 0.24$ & $3.7 \pm 1.6$ \\
\hline \multirow[t]{6}{*}{ TA-B } & $6-1275$ & $3.5+1.6$ & $8.4 \pm 3.4$ & $-0.001 \pm 0.004$ & $0.014 \pm 0.007$ & - & - & $0.7 \pm 0.2$ \\
\hline & $10-6.75$ & $3.8 \pm 1.8$ & $5.0 \pm 1.2$ & $0.002 \pm 0.002$ & $0.013 \pm 0.004$ & - & - & $0.1 \pm 0.2$ \\
\hline & $421-76$ & $10 \pm 4.0$ & $7.2 \pm 1.6$ & $-0.000 \pm 0.001$ & $0.004 \pm 0.003$ & - & - & $2.3 \pm 0.8$ \\
\hline & $10-12.76$ & $4.8 \pm 2.2$ & $3.9 \pm 1.0$ & $0.002 \pm 0.003$ & $0.012 \pm 0.004$ & - & - & - \\
\hline & $412-\pi$ & $6.1 \pm 3.0$ & $11 \pm 2.4$ & $-0.030 \pm 0.060$ & - & - & $0.35 \pm 0.06$ & $5.1 \pm 1.6$ \\
\hline & $10-1477$ & $7.0 \pm 3.0$ & $12 \pm 2.6$ & $0.001 \pm 0.004$ & $0.045 \div 0.010$ & - & - & $2.0 \pm 1.2$ \\
\hline \multirow[t]{6}{*}{ TA-49 } & $5-12.75$ & $5.2 \pm 2.2$ & $7.4 \pm 3.0$ & $0.004 \pm 0.003$ & $0.012 \pm 0.005$ & - & - & $2.1 \pm 0.8$ \\
\hline & $10-1-75$ & $20 \pm 3.6$ & $5.9 \pm 1.4$ & $-0.001 \pm 0.001$ & $0.044 \pm 0.005$ & - & - & $2.0 \pm 0.4$ \\
\hline & 421.76 & $18 \pm 4.0$ & $12 \pm 2.4$ & $-0.000 \pm 0.002$ & $0.006 \pm 0.004$ & - & - & $3.9 \pm 0.8$ \\
\hline & $10-12.76$ & $2.8 \pm 1.4$ & $6.2 \pm 1,4$ & $0.000 \pm 0.001$ & $0.027 \pm 0.006$ & - & - & - \\
\hline & $4-12.77$ & $2.6 \pm 1.2$ & $2.9+0.8$ & $0.004 \pm 0.012$ & $0.005 \pm 0.018$ & $0.51 \pm 0.10$ & $0.14 \pm 0.11$ & $4.7 \pm 2.2$ \\
\hline & $10-1477$ & $6.6+2.8$ & $11 \pm 2.6$ & $0.004 \pm 0.004$ & $0.031 \pm 0.008$ & - & - & $4.3 \pm 1.8$ \\
\hline \multirow[t]{6}{*}{ TA-33 } & $5-12-75$ & $5.0 \pm 2.0$ & $7.2 \pm 3.0$ & $-0.001 \pm 0.002$ & $0.003 \pm 0.003$ & - & - & $2.7 \pm 0.4$ \\
\hline & $10-6-75$ & $4.3 \pm 2.0$ & $5.0 \pm 1.2$ & $0.901 \pm 0.001$ & 0.00820 .003 & - & - & $0.2 \pm 0.2$ \\
\hline & $421-76$ & $7.6 \pm 3.4$ & $7.0 \pm 1.6$ & $0.002 \pm 0.001$ & $0.013 \pm 0.003$ & - & - & $2.7 \pm 0.8$ \\
\hline & $10-12.76$ & $2.5 \pm 1.2$ & $5.1 \pm 1.2$ & $0.002 \pm 0.002$ & $0.027 \pm 0.006$ & - & $0.10 \pm 2.2$ & - \\
\hline & $4-12.77$ & $8.3 \pm 3.8$ & $7.2 \pm 1.6$ & $0.001 \pm 0.010$ & $0.009 \pm 0.010$ & $0.39 \pm 0.08$ & $0.15 \pm 0.08$ & $4.5 \pm 2.0$ \\
\hline & $10-14.77$ & $7.2 \pm 3.2$ & $8.5 \pm 2.0$ & $0.003 \div 0.004$ & $0.006 \pm 0.004$ & $0.47 \pm 0.14$ & $0.06 \pm 0.22$ & $2.3 \pm 1.4$ \\
\hline
\end{tabular}


APPENDIX I

RADIOCHEMICAL ANALYSES OF SOILS SHOWING NATURAL OR WORLDWIDE-FALLOUT LEVELS (continUEd)

\begin{tabular}{|c|c|c|c|c|c|c|c|c|}
\hline & \multicolumn{7}{|c|}{$\mathrm{pCi} / \mathrm{g}$} & \multirow{2}{*}{$\begin{array}{l}\mu \mathrm{g} / \mathrm{g} \\
\text { Totai U }\end{array}$} \\
\hline & Date & Gross Alpha & Gross Beta & ${ }^{238} \mathrm{Pu}$ & $239 \mathrm{Pu}$ & ${ }^{137} \mathrm{Cs}$ & ${ }^{80} \mathrm{Sr}$ & \\
\hline \multicolumn{9}{|c|}{ Perimeter (continued) } \\
\hline \multirow[t]{8}{*}{ Frijoles } & $10.7-76$ & $4.0 \pm 2.0$ & $6.1 \pm 1.4$ & $0.001 \pm 0.002$ & $0.033 \pm 0.008$ & - & $0.50 \pm 0.20$ & - \\
\hline & 3977 & $6.7 \pm 3.0$ & $6.3 \pm 1.4$ & $0.004 \pm 0.010$ & $0.013 \pm 0.012$ & $0.79 \pm 0.12$ & $0.44 \pm 0.28$ & $14 \pm 2.4$ \\
\hline & $10-1477$ & $4.4 \pm 2.0$ & $6.5+1.6$ & $-0.001 \pm 0.002$ & $0.019 \pm 0.006$ & $0.95 \pm 0.18$ & $0.77 \pm 0.38$ & $2.6 \pm 1.6$ \\
\hline & No. of Analysis & 27 & 27 & 27 & 26 & 7 & 10 & 22 \\
\hline & Minimum & 2.5 & 3.6 & -0.030 & 0.005 & 0.39 & -0.01 & 0.1 \\
\hline & Maximum & 18 & 12 & 0.004 & 0.045 & 1.04 & 0.50 & 5.1 \\
\hline & $\bar{x}$ & 5.9 & 7.2 & 0.000 & 0.018 & 0.71 & 0.24 & 2.4 \\
\hline & s & 3.2 & 2.4 & 0.006 & 0.012 & 0.25 & 0.18 & 1.6 \\
\hline \multicolumn{9}{|l|}{ On-Site } \\
\hline \multirow[t]{6}{*}{$P M-1$} & $5-12.75$ & $5.9 \pm 24$ & $10 \pm 4.0$ & $-0.001 \pm 0.004$ & $0.015+0.009$ & - & - & $1.4 \pm 0.6$ \\
\hline & $10-6-75$ & $3.8 \pm 1.8$ & $4.8 \pm 1.2$ & $0.000 \pm 0.000$ & $0.012 \pm 0.007$ & - & - & $0.2 \div 0.2$ \\
\hline & 421.76 & $6.8 \pm 3.0$ & $0.4 \pm 1.4$ & $0.001 \pm 0.008$ & $0.009 \pm 0.009$ & - & - & $2.7 \pm 1.0$ \\
\hline & $10-12.76$ & $2.4 \pm 1.2$ & $3.5 \pm 1.0$ & $0.001 \pm 0.002$ & $0.016 \pm 0.004$ & - & $0.60 \pm 0.40$ & - \\
\hline & 412.77 & $5.3 \div 2.4$ & $7.4 \pm 1.6$ & $0.002 \pm 0.003$ & $0.011 \pm 0.004$ & $0.61 \pm 0.10$ & $0.46 \pm 0.28$ & $4.4 \pm 2.2$ \\
\hline & $10-1477$ & $6.8 \pm 3.0$ & $8.9 \pm 2.0$ & $-0.001 \pm 0.003$ & $0.017 \pm 0.006$ & $0.63+0.22$ & $0.30 \pm 0.36$ & $4.8 \pm 2.0$ \\
\hline \multirow[t]{10}{*}{ TA-33 } & $5-21 \cdot 75$ & $3.9 \pm 1.6$ & $10 \pm 4.0$ & $0.001 \pm 0.002$ & $0.003+0.007$ & - & - & $0.5 \pm 0.2$ \\
\hline & $421-76$ & $2.0 \pm 1.0$ & $1.5 \pm 0.6$ & - & - & - & - & - \\
\hline & $1 C-13-76$ & $2.3 \pm 1.2$ & $4.0 \pm 1.0$ & $0.001 \pm 0.002$ & $0.007 \pm 0.003$ & - & - & - \\
\hline & 412.77 & $15 \pm 6.0$ & $10 \pm 2.2$ & $0.003 \div 0.012$ & $0.014 \pm 0.020$ & $0.46 \pm 0.10$ & $0.10 \pm 0.02$ & $4.5 \pm 1.8$ \\
\hline & $11-10-77$ & $7.8 \pm 3.6$ & $10 \pm 2.4$ & $0.000 \pm 0.003$ & $0.011 \pm 0.006$ & $0.33 \pm 0.10$ & $0.51 \pm 0.22$ & $2.6 \pm 1.4$ \\
\hline & No. of Analysis & 11 & 11 & 10 & 10 & 4 & 5 & 8 \\
\hline & Minimum & 2.0 & 1.5 & -0.001 & 0.003 & 0.33 & 0.10 & 0.2 \\
\hline & Maximum & 15 & 10 & 0.003 & 0.017 & 0.63 & 0.60 & 4.8 \\
\hline & $\bar{x}$ & 5.6 & 7.0 & 0.001 & 0.011 & 0.51 & 0.39 & 2.6 \\
\hline & s & 3.7 & 3.1 & 0.001 & 0.004 & 0.14 & 0.20 & 1.8 \\
\hline \multicolumn{9}{|l|}{ All Soils } \\
\hline & No. of Analysis & 77 & 77 & 73 & 72 & 33 & 31 & 58 \\
\hline & Minimum & 0.1 & 1.5 & -0.030 & -0.020 & 0.07 & -0.01 & 0.1 \\
\hline & Mazimum & 18 & 12 & 0.010 & 0.045 & 1.06 & 1.60 & 5.1 \\
\hline & $\bar{x}$ & 4.9 & 6.7 & 0.000 & 0.012 & 0.52 & 0.33 & 2.1 \\
\hline & s & 3.4 & 2.4 & 0.004 & 0.012 & 0.30 & 0.27 & 1.4 \\
\hline
\end{tabular}

Note: \pm value represents twice the error term for that analysis. 
APPEND:X $\|$

RADIOCHEMICAL ANALYSES OF SEDIMENTS SHOWING NATURAL OR WORLDWIDE.FALLOUT LEVELS

pCi/g

\begin{tabular}{|c|c|c|c|c|c|c|c|c|}
\hline & Dato & Gross Alpha & Gross Bete & ${ }^{238} P_{\mathrm{PU}}$ & ${ }^{239} \mathrm{Pu}$ & ${ }_{-137}^{137}$ & ${ }^{50} \mathrm{St}_{\mathrm{P}}$ & Total U \\
\hline \multicolumn{9}{|l|}{ Regional } \\
\hline \multirow[t]{7}{*}{ Cremirs } & 7.14 .74 & $0.4 \pm 0.4$ & $5.4 \pm 0.8$ & $0.000 \pm 0.002$ & $0.001 \pm 0.002$ & $0.08 \pm 0.24$ & - & - \\
\hline & 58.75 & $1.7 \pm 0.8$ & $2.5 \pm 1.0$ & $0.002 \pm 0.003$ & $0.001 \pm 0.002$ & - & - & $0.2 \div 0.2$ \\
\hline & 0.30 .75 & $4.9 \pm 2.4$ & $3.8 \pm 1.0$ & $-0.001 \pm 0.001$ & $0.001 \pm 0.002$ & - & - & $1.4 \pm 0.8$ \\
\hline & 3.29 .76 & $9.0 \pm 4.0$ & $5.2 \pm 1.2$ & $0.000 \pm 0.601$ & $0.002 \pm 0.001$ & $0.19 \pm 0.03$ & - & $1.0 \pm 0.4$ \\
\hline & $10-476$ & $0.5 \div 0.8$ & $1.8 \pm 0.6$ & $0.000 \pm 0.001$ & $0.000 \pm 0.001$ & - & $0.01 \pm 0.14$ & - \\
\hline & 3.7 .77 & $1.6 \pm 1.0$ & $1.6 \pm 0.6$ & $0.001 \pm 0.008$ & 0.00120 .004 & $0.08 \pm 0.06$ & $-0.01 \pm 0.11$ & $0.9 \pm 1.4$ \\
\hline & $10-1877$ & 0.920 .8 & $1.7 \pm 0.8$ & $0.000 \div 0.004$ & $0.001 \pm 0.003$ & $0.06 \pm 0.06$ & $0.00 \pm 0.20$ & $1.6 \pm 1.0$ \\
\hline \multirow[t]{7}{*}{ Embudo } & 7.14 .74 & $0.7 \pm 0.4$ & $11 \pm 1.0$ & $0.001 \pm 0.004$ & $0.006 \pm 0.004$ & $0.66 \pm 0.26$ & - & - \\
\hline & 5876 & $1.6 \pm 0.8$ & $1.6 \pm 0.8$ & $-0.001 \pm 0.002$ & $0.002 \pm 0.003$ & - & - & $1.6 \pm 1.0$ \\
\hline & 10.7 .75 & $3,4 \pm 1.8$ & $3.8 \pm 1.0$ & $0.000 \pm 0.001$ & $0.011 \pm 0.004$ & - & - & $1.3 \pm 0.8$ \\
\hline & $3-28-76$ & $4.2 \pm 2.2$ & $3.5 \pm 1.0$ & $0.06 u+0.002$ & $0.002 \pm 0.002$ & $0.14 \pm C .03$ & - & $0.3 \pm 0.2$ \\
\hline & $10-4.76$ & $0.7 \pm 1.0$ & $1.6+0.8$ & $0.001 \pm 0.004$ & $0.001 \pm 0.003$ & - & $0.02 \pm 0.06$ & - \\
\hline & 3.7 .77 & $1.7 \pm 1.0$ & $1.2 \pm 0.6$ & $0.001 \pm 0.004$ & $0.002 \pm 0.008$ & $0.14 \pm 0.04$ & $-0.04 \pm 0.12$ & $2.7 \pm 2.0$ \\
\hline & $10-18.77$ & $1.5 \pm 1.0$ & $1.4 \pm 0.8$ & $-0.001 \pm 0.001$ & $0.001 \pm 0.001$ & $0.09 \pm 0.06$ & $0.77+0.27$ & $1.7 \pm 0.8$ \\
\hline \multirow[t]{7}{*}{ Orowi } & $10-1474$ & $0.4 \pm 0.2$ & $10 \quad 40.5$ & $0.000 \pm 0.000$ & $0.032 \pm 0.016$ & $0.16 \pm 0.24$ & - & - \\
\hline & $6-875$ & $1.2 \pm 0.8$ & $1.1 \pm 0.6$ & $0.000 \pm 0.000$ & $0.013 \pm 0.005$ & - & $-\cdot$ & $0.2 \pm 0.2$ \\
\hline & $10-7-75$ & $1.3 \pm 0.8$ & $1.4 \pm 0.6$ & $-0.002 \pm 0.002$ & $0.013 \pm 0.022$ & - & - & $3.8 \pm 1.2$ \\
\hline & $4-29-76$ & $3.5 \pm 2.0$ & $3.7 \pm 1.0$ & $-0.001 \pm 0.002$ & $0.002 \pm 0.002$ & $0.20 \pm 0.03$ & - & $0.5+1.0$ \\
\hline & $10-476$ & $23 \pm 1.6$ & $3.0 \pm 1.0$ & $0.000 \pm 0.002$ & $0.003 \pm 0.003$ & - & - & - \\
\hline & 3.877 & $1.7 \pm 1.0$ & $2.3 \pm 0.8$ & $0.003 \pm 0.006$ & $0.005 \pm 0.006$ & $0.06 \pm 0.06$ & $0.08 \pm 0.08$ & $0.5+1.4$ \\
\hline & $10-14.77$ & $23 \pm 1.2$ & $3.4 \pm 1.0$ & $0.000 \pm 0.002$ & $0.003 \div 0.003$ & $-0.08 \pm 0.16$ & $0.51 \pm 0.20$ & $1.3 \pm 1.0$ \\
\hline \multirow[t]{5}{*}{ Cochiti } & 7.15 .74 & $0.4 \pm 0.2$ & $4.6 \pm 0.6$ & $0.000 \pm 0.000$ & $0.000 \pm 0.000$ & $0.03 \pm 0.24$ & - & - \\
\hline & 6.8 .75 & $5.0 \pm 2.0$ & $6.4 \pm 2.6$ & $0.000 \div 0.000$ & $-0.001 \pm 0.001$ & - & - & $0.8 \pm 0.2$ \\
\hline & 10.5 .76 & $0.5+0.8$ & $1.7 \pm 0.8$ & $0.003 \pm 0.004$ & $0.002 \pm 0.003$ & - & $0.90 \pm 0.60$ & - \\
\hline & 3.277 & $3.4 \pm 1.8$ & $2.0 \pm 0.8$ & $-0.020 \pm 0.060$ & $0.030 \pm 0.040$ & $0.05+0.04$ & $0.03 \pm 0.02$ & $2.7 \pm 2.0$ \\
\hline & $10-18.77$ & $2.0 \pm 1.0$ & $1.3 \pm 0.8$ & $0.000 \pm 0.003$ & $0.001 \pm 0.002$ & 0.0410 .06 & $-0.05 \pm 0.18$ & $1.1 \pm 1.0$ \\
\hline \multirow[t]{6}{*}{ Bernolillo } & 7.16 .74 & $0.8 \pm 0.4$ & $11 \pm 1.0$ & $0.000 \pm 0.000$ & $0.004 \pm 0.002$ & $0.23 \pm 0.24$ & - & - \\
\hline & $5-875$ & $1.9 \pm 2.0$ & $2.4 \pm 1.0$ & $0.0000 \pm 0.003$ & $0.004 \pm 0.003$ & - & - & $0.6 \pm 0.2$ \\
\hline & $4 \cdot 2 \cdot 76$ & $1.1 \pm 0.6$ & $8.1 \pm 1.4$ & $-0.001 \pm 0.001$ & $0.005+0.002$ & $0.23 \pm 0.04$ & $0.80 \pm 0.20$ & $1.7 \pm 0.8$ \\
\hline & $10-5.78$ & $\therefore \varangle \pm 1.4$ & $2.6 \leq 0.3$ & $0.000-0.001$ & $0.003 \pm 0.002$ & - & $0.40 \pm 0.60$ & - \\
\hline & 3.877 & $14 \pm 8.0$ & $6.3 \pm 1.4$ & $0.001 \pm i j .004$ & $0.013 \pm 0.014$ & $0.18 \pm 0.08$ & $0.05+0.03$ & $3.4 \pm 1.6$ \\
\hline & $10-98-77$ & $1.5<1.0$ & $1.6 \pm 0.8$ & $-0.004 \pm 0.002$ & $0.001 \pm 0.002$ & $-0.01 \pm 0.12$ & $0.08+0.18$ & $2.3 \pm 1.0$ \\
\hline
\end{tabular}


APPENDIX H

RADIOCHEMICAL ANALYSES OF SEDIMENTS SHOWING NATURAL OR WORLDWIDE-FALLOUT LEVELS (cOntinUed)

\begin{tabular}{|c|c|c|c|c|c|c|c|c|}
\hline & \multicolumn{7}{|c|}{$\mathrm{pCi} / \mathrm{g}$} & \multirow{2}{*}{$\begin{array}{l}\mu \mathrm{g} / \mathrm{g} \\
\text { Total U } \\
\end{array}$} \\
\hline & Date & Gross Alpha & Gross Beta & ${ }^{238} \mathrm{Pu}_{\mathrm{u}}$ & ${ }^{239} \mathrm{Pu}$ & ${ }^{137} \mathrm{Cs}_{s}$ & ${ }^{90} \mathrm{Sr}$ & \\
\hline \multicolumn{9}{|l|}{ Regional } \\
\hline \multirow[t]{12}{*}{ Jemez } & $7 \cdot 24 \cdot 74$ & $1.0 \pm 0.4$ & $8.9 \pm 1.0$ & $0.000 \pm 0.002$ & $0.002 \pm 0.002$ & $0.34 \pm 0.24$ & - & - \\
\hline & $5-8.75$ & $1.7 \pm 0.8$ & $2.5 \pm 1 . \mathrm{C}$ & $0002 \pm 0.003$ & $0.001 \pm 0.002$ & - & - & $0.2 \pm 0.3$ \\
\hline & 9.30 .75 & $4.9 \pm 2.4$ & $3.8 \pm 1.0$ & $-0.00 \pm 0.001$ & $0.001 \pm 0.001$ & - & - & $1.4 \pm 0.8$ \\
\hline & 4.2 .76 & $10 \pm 4.0$ & $5.0 \pm 1.2$ & $0.001 \pm 0.001$ & $0.002 \pm 0.002$ & $0.13 \pm 0.03$ & - & $2.0 \pm 0.8$ \\
\hline & $10-5.76$ & $9.0 \pm 4.0$ & $6.0 \pm 1.4$ & $0.000 \pm 0.003$ & $0.008 \pm 0.004$ & - & - & - \\
\hline & 3.877 & $12 \pm 3.0$ & $4.7 \pm 1.2$ & $0.000 \pm 0.002$ & $0.006 \pm 0.004$ & $0.32 \pm 0.08$ & $0.16 \pm 0.16$ & $1.7 \pm 1.8$ \\
\hline & 10.19 .77 & $14 \pm 3.0$ & $12 \pm 2.6$ & $0.001 \pm 0.002$ & $0.007 \pm 0.004$ & $0.46 \pm 0.12$ & $0.17 \pm 0.20$ & $2.7 \pm 1.2$ \\
\hline & No. of Analysis & 39 & 38 & 39 & 39 & 23 & 17 & 27 \\
\hline & Minimum & 0.4 & 1.1 & -0.020 & -0.001 & -0.08 & -0.05 & 0.2 \\
\hline & Maximum & 14 & 12 & 0.003 & 0.032 & 0.56 & 0.80 & 3.8 \\
\hline & $\bar{H}$ & 3.3 & 4.1 & 0,000 & 0.005 & 0.16 & 0.23 & 1.5 \\
\hline & s & 3.8 & 3.0 & 0.003 & 0.007 & 0.15 & 0.32 & 1.0 \\
\hline \multicolumn{9}{|l|}{ Perimeter } \\
\hline \multirow[t]{6}{*}{ Gaule at $G-4$} & $5-12-75$ & $2.1 \pm 1.0$ & $1.6 \pm 0.8$ & $0.000 \pm 0.001$ & $0.001 \pm 0.002$ & - & - & $0.4+0.2$ \\
\hline & $10-6-75$ & $1.1 \pm 0.6$ & $1.3 \pm 0.6$ & $0.002 \pm 0.003$ & $0.002 \pm 0.002$ & - & - & $0.1 \pm 0.2$ \\
\hline & $4-21-76$ & $3.3 \pm 1.4$ & $2.9 \pm 0.8$ & $-0.006 \pm 0.007$ & $0.000 \pm 0.000$ & $0.09 \pm 0.06$ & $0.56 \pm 3.4$ & $2.7 \pm 1.0$ \\
\hline & $10-12-76$ & $1.2 \pm 0.6$ & $1,1 \pm 0.6$ & $0.000 \pm 0.002$ & $0.002 \pm 0.002$ & - & - & - \\
\hline & $4-12-77$ & $1.8 \pm 1.0$ & $1.7 \pm 0.6$ & $0.001 \pm 0.002$ & $0.000 \pm 0.003$ & -0.1310 .12 & $0.02 \pm 0.20$ & $-0.9 \pm 9.8$ \\
\hline & $10-1477$ & $1.7 \pm 1.0$ & $1.5 \pm 0.8$ & $-0.002 \pm 0.003$ & $0.001 \pm 0.002$ & $0.06 \pm 0.08$ & $0.10 \pm 0.26$ & $1.7 \pm 1.4$ \\
\hline \multirow[t]{6}{*}{ Mortandad at SR-4 } & $5-12.75$ & - & - & $0.001 \pm 0.001$ & $0.001 \pm 0.002$ & - & - & $0.3 \pm 0.2$ \\
\hline & $10-6.75$ & $1.8 \pm 1.0$ & $2.2 \pm 0.6$ & $0.000 \pm 0.0 \mathrm{v} 7$ & $-0.003 \pm 0.004$ & - & - & $0.1 \pm 0.2$ \\
\hline & 421.76 & $3.8 \pm 1.8$ & $2.4 \pm 0.8$ & $-0.002 \pm 0.005$ & $0.0+1 ;=0.006$ & $0.08+0.03$ & - & $1.4 \pm 0.8$ \\
\hline & 10.12 .76 & $2.8 \pm 1.4$ & $1.8 \pm 0.8$ & $0.002 \pm 0.002$ & $0.004 \pm 0.003$ & - & $0.11 \pm 0.06$ & - \\
\hline & $4-12.77$ & $5.1 \pm 2.4$ & $5.1 \pm 1.2$ & $0.002 \pm 0.020$ & $0.010 \pm 0.040$ & $0.01 \pm 0.16$ & $0.14 \pm 0.04$ & $2.0 \pm 1.8$ \\
\hline & $10-14 \cdot 77$ & $4.9 \pm 2.2$ & $6.5 \pm 1.6$ & $0.001 \pm 0.002$ & $0.002 \pm 0.000$ & $0.16 \pm 0.18$ & $0.14 \pm 0.22$ & $2.6 \pm 1.6$ \\
\hline \multirow[t]{6}{*}{ Pajarito at SR-4 } & 5.12 .75 & $1.4 \pm 0.8$ & $1.8 \pm 0.8$ & $0.000 \pm 0.002$ & $0.004 \pm 0.002$ & - & - & $0.1 \pm 0.2$ \\
\hline & $10-6-75$ & $2.6 \pm 1.4$ & $3.3 \pm 0.8$ & $-0.010 \pm 0.010$ & $-0.003 \pm 0.002$ & - & - & $0.2 \pm 0.2$ \\
\hline & 421.76 & $2.0 \pm 1.0$ & $1.8 \pm 0.6$ & $0.001 \pm 0.001$ & $0.000 \pm 0.001$ & $0.06 \pm 0.02$ & $-0.09 \pm 1.76$ & $1.8 \pm 0.8$ \\
\hline & $10-12.76$ & $1.7 \pm 1.0$ & $1.7 \pm 0.8$ & $0.000 \pm 0.002$ & $0.000 \pm 0.002$ & - & - & - \\
\hline & 412.77 & $9.0 \pm 4.0$ & $6.6 \pm 1.6$ & $-0.009 \pm 0.014$ & $-0.006 \pm 0.012$ & $0.15 \pm 0.08$ & $-0.06 \pm 0.12$ & $2.6 \pm 1.8$ \\
\hline & 10.1477 & $5.6 \pm 2.4$ & $6.3 \pm 1.6$ & $0.000 \pm 0.001$ & $0.009 \pm 0.004$ & $0.39+0.12$ & $0.24 \pm 0.28$ & $3.0 \pm 1.4$ \\
\hline \multirow[t]{6}{*}{ Frijoles at Pk. Hdqr. } & $5-8-75$ & $2.5 \pm 1.2$ & $2.6 \pm 1.2$ & $0.003 \pm 0.003$ & $0.001 \pm 0.002$ & - & - & $1.5 \pm 0.2$ \\
\hline & $: 0-6-75$ & $1.4 \pm 0.8$ & $1.8 \pm 0.6$ & $0.001 \pm 0.003$ & $0.000 \pm 0.001$ & - & - & $0.4 \pm 0.2$ \\
\hline & $3-30-76$ & $3.6 \pm 1.6$ & $3.5 \pm 1.0$ & $0.000 \pm 0.001$ & $0.002 \pm 0.002$ & $0.16 \pm 0.06$ & - & $2.0,50.8$ \\
\hline & $10-7.76$ & $1.4 \pm 1.0$ & $2.9 \pm 0.8$ & $0.000 \pm 0.002$ & $0.003 \pm 0.002$ & - & $0.10 \pm 0.06$ & - \\
\hline & $3-9-77$ & $1.3 \pm 0.8$ & $2.4 \pm 0.8$ & $0.005 \pm 0.012$ & $0.001 \pm 0.002$ & $-0.14 \pm 0.14$ & $0.04 \pm 0.02$ & $1.4 \pm 1.8$ \\
\hline & $10-14-77$ & $1.8 \pm 1.0$ & $1.1 \pm 0.8$ & $0.001 \pm 0.001$ & $0.002 \pm 0.002$ & $0.10 \pm 0.06$ & $0.14 \pm 0.22$ & $3.0 \pm 1.2$ \\
\hline
\end{tabular}


APPENDIX II

RADIOCHEMICAL ANALYSES OF SEDIMENTS SHJWING NATURAL OR WORLDWIDE-FAI.LOUT LEVELS (CONTInUEd)

\begin{tabular}{|c|c|c|c|c|c|c|c|}
\hline \multicolumn{7}{|c|}{ pCi,ós } & \multirow{2}{*}{$\begin{array}{l}\text { mog/g } \\
\text { Total U }\end{array}$} \\
\hline Dare & Gros Alpha & Gross Beta & ${ }^{230} \mathrm{Pu}$ & ${ }^{239} \mathrm{Fu}$ & ${ }^{137} \mathrm{Cs}$ & ${ }^{90} \mathrm{~s}$ & \\
\hline No. of Anstryis & 23 & 23 & 24 & 24 & 12 & 12 & 20 \\
\hline Minimum & 1.1 & 1.1 & -0.010 & -0.008 & -0.14 & -0.09 & -0.9 \\
\hline Maximum & 8.0 & o..6 & 0.005 & 0.010 & 0.39 & 0.56 & 3.0 \\
\hline $\bar{x}$ & 2.8 & 2.8 & 0.000 & 0.002 & 0.08 & 0.12 & 1.3 \\
\hline 6 & 1.9 & 1.7 & 0.003 & 0.003 & 0.14 & 0.17 & 1.2 \\
\hline
\end{tabular}

\begin{tabular}{|c|c|c|c|c|c|c|c|c|}
\hline \multicolumn{9}{|l|}{ On-Sito } \\
\hline \multirow[t]{5}{*}{ Whter at Beta } & $5-20-75$ & $2.2 \pm 1.0$ & $2.2 \pm 1.0$ & $0.000 \div 0.002$ & $0.001 \pm 0.002$ & - & - & $0.8 \pm 0.4$ \\
\hline & $9-26-75$ & $0.6 \pm 0.4$ & $0.2 \pm 0.4$ & $0.000 \pm 0.001$ & $0.002 \pm 0.002$ & - & - & $0.2 \pm 0.4$ \\
\hline & 4.21 .76 & $2.6 \pm 1.2$ & $1.8 \pm 0.6$ & $0.000 \pm 0.002$ & $0.003 \div 0.002$ & $0.11 \pm 0.03$ & - & $0.3 \pm 0.6$ \\
\hline & 411.77 & $10 \pm 4.0$ & $4.5 \pm 1.2$ & $0.001 \pm 0.003$ & $0.000 \pm 0.003$ & $0.19 \pm 0.04$ & $-0.11 \pm 0.12$ & $4.1 \pm 2.4$ \\
\hline & $11-19-77$ & $3.4 \pm 1.6$ & $5.1 \pm 1.4$ & $-0.002+0.003$ & $0.011 \pm 0.004$ & $0.80 \pm 0.14$ & - & $3.0 \times 1.6$ \\
\hline \multirow[t]{6}{*}{ Water at SR-4 } & 5.12 .76 & $2.4 \pm 1.0$ & $2.6 \pm 1.2$ & $000 \pm 0.002$ & $0.001 \pm 0.001$ & - & - & $0.3 \pm 0.2$ \\
\hline & $10-6-75$ & $2.4 \pm 1.2$ & $3.3 \pm 0.8$ & $0.001 \pm 0.008$ & - & - & - & $0.1 \pm 0.2$ \\
\hline & 421.76 & $8.7 \pm 3.8$ & $6.4 \pm 1.4$ & $0.002 \times 0.002$ & $0.006 \pm 0.003$ & $0.39 \pm 0.04$ & $0.36 \pm 2.1$ & $3.7 \pm 0.8$ \\
\hline & $10-12-76$ & $1.3 \pm 0.8$ & $1.2+0.6$ & $0.001 \pm 0,003$ & $0.001 \pm 0.003$ & - & $0.06 \pm 0.10$ & - \\
\hline & 4.12 .77 & $2.1 \pm 1.0$ & $2.8 \pm 0.8$ & $-0.00 ; 00.01 \%$ & $0.010+0.016$ & $0.14 \pm 0.06$ & $0.28 \pm 0.16$ & $1.8+1.6$ \\
\hline & $10-1477$ & $6.1 \pm 2.8$ & $13 \pm 2.8$ & $-0.000 \pm 0.002$ & $0.036 \pm 0.006$ & - & $0.46 \pm 0.24$ & $3.3 \pm 1.4$ \\
\hline \multirow[t]{10}{*}{ Ancho at SR-4 } & 421.76 & $9.0 \pm 4.0$ & $6.9 \pm 1.6$ & $0.000 \pm 0.000$ & $0.005 \pm 0.003$ & $0.17 \pm 0.03$ & - & $3.1 \pm 0.8$ \\
\hline & 10.12 .76 & $1.2 \pm 0.6$ & $2.0 \pm 0.8$ & $0.000 \pm 0.003$ & $0.001 \pm 0.003$ & - & $0.20 \pm 0.26$ & - \\
\hline & 412.77 & - & - & $0.00\} \pm 0.012$ & $-0.010 \div 0.006$ & $0.14 \pm 0.08$ & $0.04 \pm 0.08$ & $2.9 \pm 2.0$ \\
\hline & $10-14.77$ & $3.8 \pm 1.8$ & $5.8 \pm 1.4$ & $0.00 / 40.002$ & $0.008 \div 0.004$ & $0.68 \Varangle 0.18$ & $0.30 \pm 0.20$ & $2.1 \pm 1.6$ \\
\hline & No. of Analyis & 14 & 14 & 15 & 14 & 8 & 8 & 13 \\
\hline & Minimum & 0.6 & 0.2 & -0.002 & -0.010 & 0.11 & -0.11 & 0.1 \\
\hline & Naximum & 10.0 & 13.0 & 0.002 & 0.026 & 0.80 & 0.46 & 4.1 \\
\hline & $x$ & 4.0 & 4.1 & 0.000 & 0.005 & 0.33 & 0.20 & 2.0 \\
\hline & 3 & 3.1 & 3.3 & 0.001 & 0.010 & 0.27 & 0.19 & 1.5 \\
\hline & & & & 60 & & & & \\
\hline \multicolumn{9}{|l|}{ All Sodiments } \\
\hline & No. of Analyes & 76 & 76 & 78 & 77 & 43 & 37 & 60 \\
\hline & Minimum & 0.4 & 0.2 & -0.020 & -0.010 & -0.14 & -0.11 & -0.8 \\
\hline & $\underline{\text { Maximum }}$ & 14.0 & 13.0 & 0.005 & 0.036 & 0.80 & 0.90 & 4.1 \\
\hline & $\bar{x}$ & 3.3 & 3.7 & 0.000 & 0.004 & 0.17 & 0.20 & 1.5 \\
\hline & s & 3.2 & 2.8 & 0.003 & 0.007 & 0.19 & 0.25 & 1.2 \\
\hline
\end{tabular}

Note: \pm values represent twice the error term for that analysis. 
APPENDIX III

RADIOCHEMICAL ANALYSES OF SOIL FROM EFFLUENT RELEASE AREAS

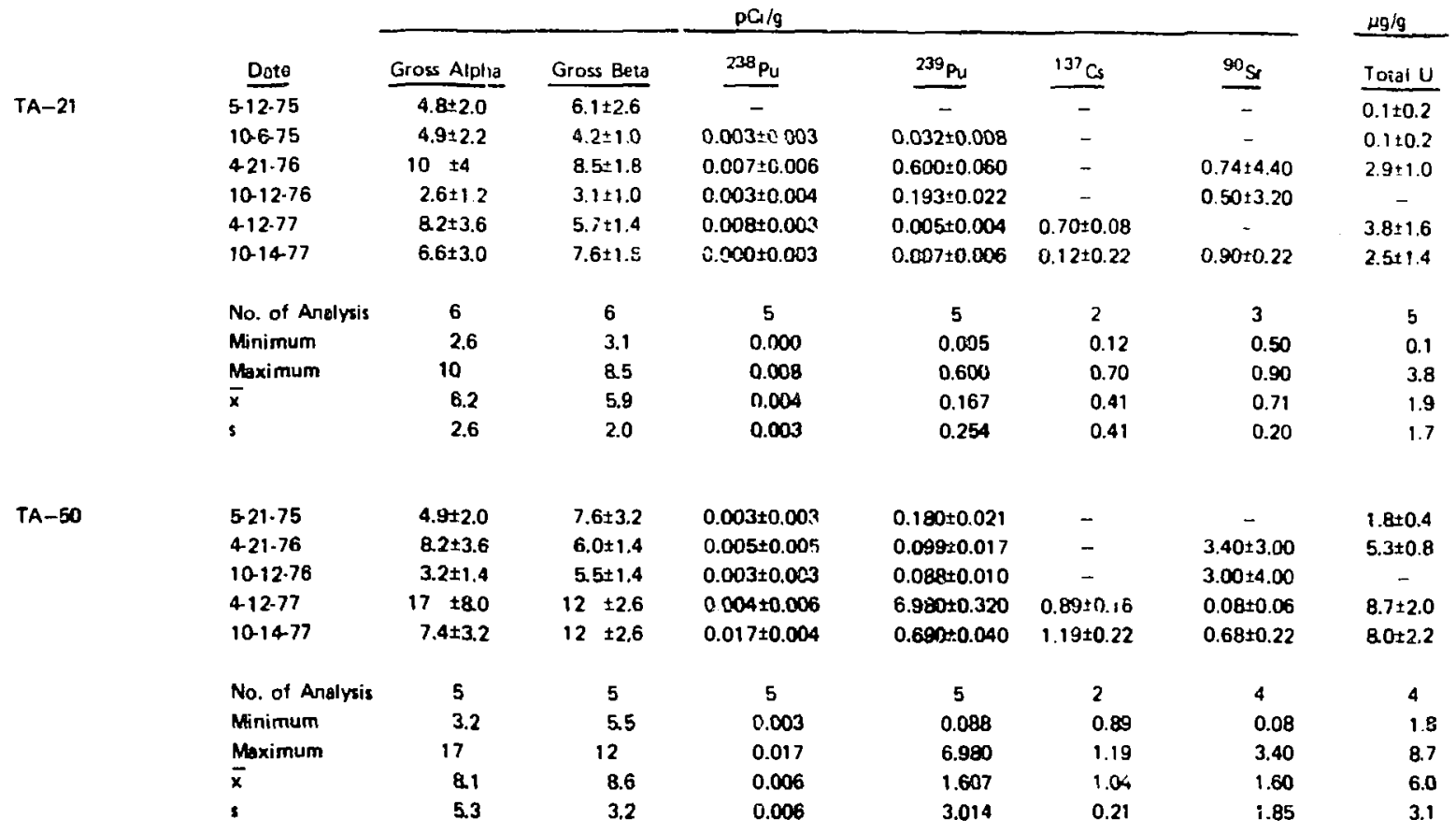

Note: \pm values ropresent twico the error term for that analysis. 
APPENDIX IV

AADIOCHEMICAL ANALYSES OF SEDIMENTS FROM EFFLUENT RELEASE AREAS

\begin{tabular}{|c|c|c|c|c|c|c|c|c|}
\hline & \multicolumn{7}{|c|}{$\mathrm{pCs} / \mathrm{g}$} & \multirow{2}{*}{$\begin{array}{l}\text { H/g } \\
\text { Totol U } \\
\end{array}$} \\
\hline & Dante & Gross Alpha & Gross Boto & $230_{p_{u}}$ & $\underline{200} \mathrm{Pu}_{\mathrm{Pu}}$ & ${ }^{137} a$ & ${ }^{90} \mathrm{Sr}$ & \\
\hline \multirow[t]{11}{*}{ Auebio as SR-A } & $6-12.75$ & $2.2+1.0$ & $1.2 \pm 0.6$ & $0.002+0.003$ & $0.352+0.034$ & - & - & $1.3 \pm 0.4$ \\
\hline & 10.6 .75 & $1.0 \pm 0.8$ & $0.6>0.4$ & $0.001 \pm 0.002$ & $0.274 \pm 0.026$ & - & - & $0.1 \pm 0.2$ \\
\hline & $4-21.78$ & $28 \pm 8.0$ & $28 \pm 6.0$ & $0.000 \pm 0.001$ & $0.011 \pm 0.003$ & $0.46 \pm 0.04$ & - & $30 \pm 4.0$ \\
\hline & $10-12.76$ & $0.8 \pm 0.6$ & $0.6 \pm 0.6$ & $0.002 \pm 0.002$ & $0.355+0.034$ & - & $0.20 \pm 0.18$ & - \\
\hline & 411.77 & $2.3 \pm 1.0$ & $1.8 \pm 0.6$ & $.002 \pm 0.010$ & $0.380 \pm 0.050$ & $0.07 \pm 0.04$ & $0.09 \pm 0.18$ & $3.1 \pm 1.8$ \\
\hline & $10-1477$ & $2.3 \pm 1.2$ & $2.8 \pm 0.8$ & $0.003 \pm 0.006$ & $0.228 \pm 0.030$ & $0.15+0.08$ & $-0.04 \div 0.18$ & - \\
\hline & No. of Anstyis & 6 & 6 & 6 & 6 & 3 & 3 & 4 \\
\hline & Afinimum & 0.8 & 0.6 & 0.000 & 0.011 & 0.07 & -0.04 & 0.1 \\
\hline & Mximum & 21 & 28 & 0.003 & 0.360 & 0.48 & 0.20 & 30 \\
\hline & $\bar{x}$ & 4,9 & 5.7 & 6.002 & 0.267 & 0.23 & 0.08 & 66 \\
\hline & s & 7.9 & 10.8 & 0.001 & 0.138 & 0.21 & 0.12 & 14 \\
\hline \multirow[t]{11}{*}{ Los Alamos at TW-3 } & 418.75 & $2.7 \pm 1.2$ & $2.1 \pm 0.6$ & $0.002 \pm 0.002$ & $0.0 \% 6+0.012$ & - & - & $1.1+0.2$ \\
\hline & $10-6-75$ & - & - & $0.032 \pm 0.003$ & $0.108 \pm 0.011$ & - & - & - \\
\hline & $4-21.76$ & $26 \pm 1.2$ & $28 \pm 6.0$ & $0.059 \pm 0.008$ & $0.214 \pm 0.016$ & $0.16 \pm 0.10$ & $2.5 \pm 2.8$ & $1.4 \pm 0.6$ \\
\hline & $10-13-76$ & $8.4 \pm 0.8$ & $19 \pm 3.8$ & $0.115 \pm 0.016$ & $0.720 \pm 0.060$ & $0.91 \pm 0.08$ & $3.9 \pm 0.4$ & - \\
\hline & $4-11-77$ & $2.7 \pm 1.4$ & $\infty \pm 6.0$ & $0.099 \pm 0.022$ & $0.040 \div 0.060$ & $26 \pm 3.6$ & $2.4 \pm 0.28$ & $1.2 \pm 1.6$ \\
\hline & $10-18-77$ & $3.9 \pm 1.8$ & $52 \pm 10$ & $0.111 \pm 0.012$ & $0.488-0.030$ & $28 \pm 3.6$ & $4.9 \pm 0.40$ & $3.0 \pm 1.4$ \\
\hline & No. of Anslris & 5 & 5 & 6 & $\mathbf{6}$ & 4 & 4 & 4 \\
\hline & Minimum & 1.4 & 2.1 & 0.002 & 0.040 & 0.16 & 2.4 & 1.1 \\
\hline & Maximum & 3.8 & 52 & 0.115 & 0.720 & 26 & 8.5 & 3.0 \\
\hline & $\bar{x}$ & 2.7 & 26 & 0.070 & 0.274 & 13 & 4.8 & 1.7 \\
\hline & $s$ & 0.9 & 18 & 0.046 & 0.272 & 15 & 2.6 & 0.9 \\
\hline \multirow[t]{11}{*}{ Los Alamon of SR $\rightarrow$} & $7-1.75$ & $1.5 \pm 0.6$ & $4.4 \pm 1.0$ & $0.008 \pm 0.004$ & $0.163 \div 0.016$ & - & - & $0.3 \pm 0.2$ \\
\hline & 106.75 & $0.9 \pm 0.6$ & $2.9 \pm 0.8$ & $0.006 \pm 0.012$ & $0.046 \pm 0.009$ & - & - & $0.1 \pm 0.2$ \\
\hline & 421.78 & $4.6 \pm 2.0$ & $5.0 \pm 1.2$ & $0.001 \pm 0.002$ & $0.033 \pm 0.006$ & $1.6 \pm 0,10$ & - & $3.3+0.8$ \\
\hline & 10.12 .76 & $1.1 \pm 0.6$ & $1.6 \pm 0.8$ & $0.003+0.002$ & $0.022 \pm 0.006$ & $0.88 \pm 0.08$ & $0.41 \geq 0.10$ & - \\
\hline & $4-11-77$ & $1.8 \pm 1.0$ & $3.7 \pm 1.0$ & $-0.003 \pm 0.010$ & $0.021 \pm 0.012$ & $0.67 \pm 0.12$ & $0.05 \pm 0.03$ & $3.6 \pm 2.2$ \\
\hline & $10-1471$ & $2.6 \pm 1.2$ & $4.0 \pm 1.2$ & $0.002 \pm 0.003$ & $0.022 \pm 0.006$ & $0.72 \pm 0.24$ & $0.40 \pm 0.26$ & $3.5 \pm 1.4$ \\
\hline & No. of Analysis & 6 & 6 & 6 & 8 & 4 & 3 & 5 \\
\hline & Minimum & 0.9 & $1 . \overline{5}$ & -0.003 & 0.021 & 0.67 & 0.05 & 0.9 \\
\hline & Maximum & 4.6 & 5.0 & 0.008 & 0.163 & 1.5 & 0.41 & 3.6 \\
\hline & $\bar{x}$ & 2.1 & 3.6 & 0.003 & 0.051 & 0.82 & 0.28 & 2.2 \\
\hline & 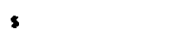 & 1.4 & 1.2 & 0.004 & 0.055 & 0.38 & 0.21 & 1.8 \\
\hline
\end{tabular}


AFPENDIX IV

RADIOCHEMICAL ANALYSES OF SEDIMENTS FROIM EFFLUENT RELEASE AREAS (continUed)

\begin{tabular}{|c|c|c|c|c|c|c|c|c|}
\hline & \multirow[b]{2}{*}{ Date } & \multicolumn{6}{|c|}{$\underline{\mathrm{pCi} / \mathrm{g}}$} & \multirow{2}{*}{$\frac{\mu g / g}{\text { Toral U }}$} \\
\hline & & Gross Alpha & Gross Beta & $238_{9 u}$ & ${ }^{239} \mathrm{Pu}$ & ${ }^{837} \mathrm{Cs}$ & ${ }^{90} \mathrm{Sr}$ & \\
\hline \multirow[t]{10}{*}{ Mortandad at TW-8 } & $4.18-75$ & - & - & $4.070 \pm 0.220$ & $0.950 \pm 0.080$ & - & - & - \\
\hline & $10-6-75$ & - & - & $4.970 \pm 0.240$ & $1.180 \pm 6.090$ & - & - & - \\
\hline & $10-15-76$ & - & - & $4.670 \times 0.220$ & $0.890 \pm 0.080$ & - & - & - \\
\hline & $4-13-77$ & $7.1 \pm 3.0$ & $67 \pm 14$ & 4.42010 .100 & $0.940 \pm 0.036$ & $71 \pm 10$ & $2.7 \pm 0.4$ & $3.4 \pm 1.8$ \\
\hline & $11 \cdot 17.77$ & $8.4 \pm 3.6$ & $75 \pm 16$ & $6.010 \pm 0.300$ & $15.600 \times 0.600$ & $71 \pm 10$ & - & $1.0 \pm 4.0$ \\
\hline & No. of Analysis & 2 & 2 & 5 & 5 & 2 & 1 & 2 \\
\hline & Minimum & 7.1 & 67 & 4.070 & 0.953 & - & - & 1.0 \\
\hline & Maximum & 8.4 & 75 & 6.010 & 15.600 & 71 & 2.7 & 3.4 \\
\hline & $\bar{x}$ & 7.8 & 71 & 4.828 & 3.912 & 71 & - & 2.2 \\
\hline & s & 0.9 & 5.7 & 0.739 & 6.535 & 0 & - & 1.7 \\
\hline \multirow[t]{10}{*}{ Portilio at TA-36 } & $5-21 \cdot 75$ & $3.5+1.6$ & $5.6 \pm 2.4$ & $0.004 \pm 0.003$ & $0.005+0.003$ & - & - & $0.7 \pm 0.2$ \\
\hline & $421-76$ & $10 \pm 4.0$ & $6.9 \pm 1.6$ & $-0.002 \pm 0.001$ & $0.005+0.002$ & $0.33 \pm 0.04$ & - & $2.8 \pm 0.8$ \\
\hline & $10-13-76$ & $3.6 \pm 1.6$ & $11 \pm 2.4$ & $0.001 \pm 0.003$ & $0.005 \pm 0.004$ & - & - & - \\
\hline & 412.77 & $6.8+3.0$ & $5.1 \pm 1.2$ & $0.000 \pm 0.022$ & $0.002 \pm 0.002$ & $0.10 \pm 0.06$ & $0.04 \pm 0.02$ & $5.6 \pm 1.8$ \\
\hline & 11.22 .77 & $12 \pm 4.0$ & $15 \pm 3.2$ & $0.000 \pm 0.002$ & $0.006 \pm 0.003$ & $0.30 \pm 0.22$ & $0.16 \pm 0.20$ & $5.1 \pm 1.8$ \\
\hline & No. of Analysis & 5 & 5 & 5 & 5 & 3 & 2 & 4 \\
\hline & Minimum & 3.5 & 5.1 & -1.002 & 0.002 & 0.10 & 0.04 & 0.7 \\
\hline & Maximum & 12 & 15 & 0.004 & 0.006 & 0.33 & 0.16 & 5.6 \\
\hline & $\bar{x}$ & 7.2 & 8.7 & 0.001 & 0.005 & 0.24 & 0.10 & 3.6 \\
\hline & : & 3.8 & 4.2 & 0.002 & $0.00 ?$ & 0.13 & 0.68 & 2.3 \\
\hline
\end{tabular}

Note: + values represent twice the error term for that analysis. 